\title{
Complementary Visualization of Mitotic Barley Chromatin by Field-Emission Scanning Electron Microscopy and Scanning Force Microscopy
}

\author{
A. Schaper, ${ }^{* 1}$ M. Rößle, $\dagger$ H. Formanek, $\dagger$ T. M. Jovin, ${ }^{*}$ and G. Wanner ${ }^{\dagger}, 2$ \\ *Department of Molecular Biology, Max Planck Institute for Biophysical Chemistry, 37070 Göttingen, Germany; \\ and $†$ Botanical Institute of LMU-Munich, Menzingerstrasse 68, 80638 Munich, Germany
}

Received June 26, 1999, and in revised form November 12, 1999

The surface structure of mitotic barley chromatin was studied by field-emission scanning electron microscopy (FESEM) and scanning force microscopy (SFM). Different stages of the cell cycle were accessible after a cell suspension was dropped onto a glass surface, chemical fixed, and critically point dried. Imaging was carried out with metal-coated specimen or uncoated specimen (only for SFM). The spatial contour of the chromatin could be resolved by SFM correlating to FESEM data. The experimentally determined volume of the residue chromatin during mitosis was within the range of $65-85 \mu \mathrm{m}^{3}$. A comparison with the theoretically calculated volume indicated a contribution of about $40 \%$ of internal cavities. Decondensation of chromosomes by proteinase $K$ led to a drastic decrease in the chromosome volume, and a 3-D netlike architecture of the residue nucleoprotein material, similar to that in the intact chromosome, was obvious. Incubation of metaphase chromosomes in citrate buffer permitted access to different levels of chromatin packing. We imaged intact chromosomes in liquid by SFM without any intermediate drying step. A granular surface was obvious but with an appreciably lower resolution. Under similar imaging conditions proteinase $K$-treated chromosomes exhibited low topographic contrast but were susceptible to plastic deformations. $\odot 2000$ Academic Press

Key Words: AFM; cell cycle; plant chromosome; DNA; scanning electron microscopy; scanning force microscopy; SFM.

\section{INTRODUCTION}

The structural integrity and functional repertoire of genomic DNA in vivo are maintained by the

\footnotetext{
${ }^{1}$ Present address: Stephanusstrasse 2, D-30449 Hannover, Germany.

2 To whom correspondence should be addressed.
}

controlled condensation of DNA-protein assemblies throughout the cell cycle. Many efforts have been undertaken to resolve the structural details at the different levels of chromatin condensation using electron microscopy (EM), crystallography, spectroscopy, and molecular biological techniques (for a review see van Holde, 1988). Although the higher order structural hierarchy of chromatin organization, in terms of the folding of the elementary fibril (nucleosomal chain) into fibrous structures of distinct sizes, has received much attention over the past two decades, the different levels of chromatin organization have hardly begun to be elucidated (for review see Cook, 1995). Several preparation techniques have been elaborated for rendering the chromatin accessible to structural investigations by highresolution microscopy (for a review see Zentgraf et al., 1987). Scanning electron microscopy (SEM) can provide valuable data about the three-dimensional (3-D) organization of the chromatin and different levels of condensation (Harrison et al., 1982, 1987; Mullinger and Johnson, 1987; Rattner and Lin, 1987; Sumner, 1991; Wanner et al., 1991; Pelling and Allen, 1993; Takayama and Hiramatsu, 1993; Rizzoli et al., 1994). Since FESEM is limited to the investigation of dried samples, it is crucial that the sample preparation procedure preserve the native structure of the biomaterials. Martin et al. (1994, 1996) have introduced the drop preparation technique that yielded well-preserved structures of mitotic, meiotic, or interphase chromatin suitable for high-resolution SEM. Scanning force microscopy (SFM) permits the structural resolution of the topography of biological material adsorbed at air-solid or liquid-solid interfaces with up to subnanometer resolution. The advantage of the SFM for biologists is that it can visualize nonconductive materials in a nonvacuous (i.e., air or liquid) environment. In effect, fixation, contrast enhancement, and labeling are not required. The SFM was successfully applied in the investigation of the 
nucleosomal chain morphology (Allen et al., 1993; Vesenka et al., 1992; Fritzsche et al., 1994a; Leuba et al., 1994), of chromatin fibers in air and in liquid (Fritzsche et al., 1994b, Fritsche and Henderson, 1996), and of the overall structure of air-dried and rehydrated metaphase chromosomes (De Grooth and Putman, 1992; De Grooth et al., 1992; Putman et al., 1993; Rasch et al., 1993; McMaster et al., 1994).

In this study we have investigated different states of barley chromatin condensation during mitosis by means of FESEM and SFM in air-vacuum and in liquid. We show SFM images of different condensation states of the chromatin, indicating that the drop technique is of general use for the preparation of chromatin free of any surface contaminations and for chromosome decondensation assays.

\section{MATERIALS AND METHODS}

All reagents were of analytical grade and were from Serva (Heidelberg, FRG), if not otherwise stated. We used doubledistilled (quartz glass distillery) and ultrafiltered (Amicon) water in the preparation of all buffer/salt solutions. Glass slides with laser engraved locator grids (Laser Marking, Fischen, FRG) were used after they were cleaned in chromic acid.

Preparation of chromatin samples. Seedings of barley (Hordeum vulgare, Steffi) were germinated on moist filter paper in a wet chamber for 3 days at $4^{\circ} \mathrm{C}$ in the dark and subsequently incubated for $8 \mathrm{~h}$ at $20^{\circ} \mathrm{C}$. For synchronization of the meristematic cells in the S-phase of the cell cycle the roots were incubated for 18 $\mathrm{h}$ in $1.25 \mathrm{M}$ hydroxyurea. For arresting the cell cycle in metaphase, roots were incubated in $4 \mu \mathrm{M}$ amiprophosmethyl (Bayer, Leverkusen, FRG) for $4 \mathrm{~h}$ at $20^{\circ} \mathrm{C}$ and washed $3 \times$ with water (Dolezel et al., 1992; Busch et al., 1994). Root tips were chopped, fixed in ethanol/acetic acid (fixative I, 3/1 (v/v)), and stored at $-20^{\circ} \mathrm{C}$. The meristematic tissue of the root tips was digested in a mixture of $10 \%$ cellulase "Onozuka" R10 and $10 \%$ pectolyase Y-23 (Kikkoman, Düsseldorf, FRG) in $75 \mathrm{mM} \mathrm{KCl,} 7.5 \mathrm{mM}$ EDTA, pH 4.5 , for $90 \mathrm{~min}$ at $30^{\circ} \mathrm{C}$. The cell suspension was filtered through a nylon mesh ( $80-\mu \mathrm{m}$ mesh size) and treated with hypotonic $\mathrm{KCl}$ (75 $\mathrm{mM}$ ) for $5 \mathrm{~min}$ at $20^{\circ} \mathrm{C}$. The nuclei were spun down for $7 \mathrm{~min}$ at $80 \mathrm{~g}$, resuspended in fixative I, and washed and pelleted $3 \times$ in fixative I. Finally, nuclei were stored in fixative I at $-20^{\circ} \mathrm{C}$. Chromosomes were prepared according to the drop technique of Martin et al. (1996). Briefly, an aliquot of the nuclei suspension was dropped on ice-cold glass slides. After a drop of $45 \%$ acetic acid was added and just before the drop was evaporated the area was covered with a coverslip, gently squashed, and incubated for 30 min on dry ice. Finally, the coverslip was removed and the glass slide was incubated for $45 \mathrm{~min}$ in fixative II (2.5\% glutaraldehyde in $75 \mathrm{mM}$ cacodylate (Merck, Darmstadt, FRG), $2 \mathrm{mM} \mathrm{MgCl}_{2}, \mathrm{pH}$ 7). For FESEM and SFM of dried specimens, samples were dehydrated in ascending concentrations of acetone (20, 40, 60, 80, $100 \%(\mathrm{v} / \mathrm{v}))$ and critical point dried (CPD) (Critical Point Dryer, Balzers, Liechtenstein) using $\mathrm{CO}_{2}$ as the transition fluid. For SFM imaging in liquid the glass slide was transferred to water and mounted to the BioScope. For mounting samples to the NanoScope the glass slides were cut into pieces $\leq 15 \mathrm{~mm}$ in diameter.

Controlled decondensation of metaphase chromosomes. Decondensation assays were performed with metaphase chromosome samples prepared by the drop technique. Enzymatic degradation of chromosomes was carried out with proteinase $K$. Glass slides were fixed in $2.5 \%$ glutaraldehyde/75 mM cacodylate buffer, $\mathrm{pH} 7$, for $1 \mathrm{~h}$, washed $3 \times$ for $15 \mathrm{~min}$ in water, and subsequently incubated with proteinase $K(0.1 \mathrm{mg} / \mathrm{ml}), 10 \mathrm{mM}$ Tris- $\mathrm{HCl}, \mathrm{pH} 7$, for $2 \mathrm{~h}$ at $37^{\circ} \mathrm{C}$. After proteinase treatment glass slides were washed $3 \times(15$-min incubation for each step) in water. Samples were incubated in fixative II for $2 \mathrm{~h}$ and again washed $3 \times$ followed by CPD. For imaging in liquid the CPD step was omitted. For chemical decondensation the metaphase chromosomes were incubated in $60 \mathrm{mM}$ sodium citrate, $\mathrm{pH} 7.2$, for $1 \mathrm{~h}$ at $20^{\circ} \mathrm{C}$ according to a procedure of Marsden and Laemmli (1979). Samples were washed twice with water prior to fixation in $2.5 \%$ glutaraldehyde in $10 \mathrm{mM}$ Tris-HCl buffer, $\mathrm{pH}$ 7.2. Again samples were washed twice in water and were critical point dried. Light microscopy (phase and differential interference contrast) was applied for the preselection of chromatin-covered surface regions facilitated by the locator grid engraved in the glass slide (cf. Martin et al., 1996). The latter also enabled localization of the same region in the FESEM and in the SFM.

Field emission scanning electron microscopy. For FESEM we used a Hitachi S-4100 equipped with an Autrata YAG-type detector for back-scattered electrons (BSE). Samples were coated with 2-3 nm Au/Pd (80/20) with a magnetron sputter-coater (050 SCD, Balzers, LIE) and examined at various voltages.

Scanning Force Microscopy. SFM measurements of CPD dried specimen were performed with a NanoScope IIIa-multimode SPM (Digital Instruments (DI), Santa Barbara, CA). In some cases a sticky tape was applied to the dry sample surface prior to SFM imaging (Pietrasanta et al., 1994, 1996). The SFM was operated under ambient conditions at $18-27^{\circ} \mathrm{C}$ and at a relative humidity in the range of $20-45 \%$. Scanning was carried out with a Jscanner with a $135 \times 135 \times 5(x, y, z) \mu^{3}$ scan range. All images were taken at a line scan rate of $\sim 4 \mathrm{~Hz}$. Investigations with the NanoScope in liquid were conveyed with a liquid measuring chamber (DI). Alternatively, for imaging in liquid we used a BioScope (DI). Surface profiling with the NanoScope and the BioScope was in permanent contact mode and in tapping mode (only for imaging in liquid with the NanoScope equipped with an Ultralever). We used different types of microfabricated scanning tips integrated into triangular cantilevers with spring constants of $\sim 0.2 \mathrm{~N} / \mathrm{m}$ : (i) microfabricated pyramidal shaped $\mathrm{Si}_{3} \mathrm{~N}_{4}$ - tips (DI) with a typical tip radius of $20-50 \mathrm{~nm}$, (ii) conical shaped Si-tips (Ultralever, Park Scientific Instruments, Sunnyvale, CA) with a typical tip radius of $3-5 \mathrm{~nm}$, and (iii) electron beam deposited (EBD) tips of $\sim 1.5 \mu \mathrm{m}$ in length and with a tip radius in the range of $5-10 \mathrm{~nm}$ as specified by the supplier (Hart Probe, Materials Analytical Services, Raleigh, NC). EBD tips were deposited on top of a pyramidal shaped $\mathrm{Si}_{3} \mathrm{~N}_{4}$ tip from DI. The standard planefit correction (first-order fits) of the NanoScope software was applied to the data. The various images were combined and processed for presentation with the programs Photoshop (Adobe Systems) and Canvas (Deneba Systems) for the Apple Macintosh computer. Cross-sectional analyses were performed with the NanoScope software. The reported lateral dimension of a surface feature is the full width at half-maximum height. For image processing we used ImageSXM software (Barrett, 1997). For volume determinations (see also Fritzsche and Henderson, 1996; Allen et al., 1996) a mean background value was subtracted from the image data. Structural features were selected by applying a binary mask generated from thresholding the surface corrugations. Residue surface contaminations were erased from the binary mask. The mask was overlaid on the original image and used to extract those data points that comprised the chromatin. The chromatin volume was calculated by collecting the volumes of the data points (voxels) within the mask. For the determination of the mean height of condensed chromatin a similar procedure was applied to the data. The upper $10^{4}$ pixel values within the masked chromosome domain were collected for calculation of the mean height and standard deviation. 


\section{RESULTS}

\section{The Apparent Volume of the Residue Chromatin Is Constant during Mitosis}

Figures $1 \mathrm{~A}-1 \mathrm{~F}$ present SFM images of the chromatin of the cell cycle separated by digital image processing, i.e., background subtraction, thresholding, and binary filtering. The chromatin in all phases appeared well distinguished from the basal plane and free from any particle contaminations. Different morphologies that were typical for the distinct phases of mitosis were obvious, including the amorphous mass of interphase chromatin (Fig. 1A), the condensed states from prophase to metaphase (Figs. 1C, and 1D) comprising 14 chromosomes in barley, and the redistribution of the chromatin between mother and daughter cells in anaphase and telophase (Figs. $1 \mathrm{E}$ and $1 \mathrm{~F})$. For comparison, the corresponding FESEM micrographs of the anaphase and telophase are shown in Figs. $1 \mathrm{H}$ and 1J. The chromatin in metaphase (Fig. 1D) is more condensed by about one order of magnitude compared to its interphase appearance (Fig. 1A) as calculated from the chromatin covered surface area per unit height. The latter is the mean height of the chromatin domain. In prophase the condensation process started from the centromeric region presumably by aggregation/ transformation of the granular interphase chromatin into chromomers, which then condensed to form a cylindrical body. In this state the chromatids were often discernible from a topographic point of view even in the uncondensed telomeric region during prometaphase (Martin et al., 1996). Parallel arrangement of fibers, which is also a characteristic feature predominantly seen in prophase chromatin by FESEM (Martin et al., 1996), was not resolved by SFM. Prophase chromosomes separated into pairs of chromatids in late prophase or early metaphase. During subsequent chromatin condensation in prometaphase and metaphase, the limitations of instrumental resolution caused by finite SFM tip size were obvious and multiple images of the tip face at steep chromosome edges were perceptible (data not shown, see Discussion). In anaphase (Fig. 1E) decondensation proceeded from the telomeric region and an amorphous chromatin structure was visible in telophase (Fig. 1F). The total volume of the resident part of each phase during mitosis was determined by means of image processing. From the diagram in Fig. $1 \mathrm{G}$ it is evident that the apparent volume of the residue chromatin during mitosis is within the range of $65-85 \mu^{3}$. The slightly higher volume in anaphase is mainly due to the overlap of chromosomes, thereby forming gaps not accessible to the SFM tip, and thus led to an additional contribution to the apparent volume. Thus we can conceive that the volume is nearly constant within the range of errors independent of the cell and of the mitosis phase.

In Fig. 2 representative SFM images of the ultrastructure of chromatin are presented that exhibit the most prominent morphological changes of the chromatin during the cell cycle on the nanometer scale. In interphase (Figs. 2A, and 2B) a globular morphology was the common structural motif with an apparent globule diameter of about 100-300 nm. In prometaphase the chromosomal surface was densely packed with granules and condensation of chromatin was accompanied by a drastic increase in their size (Figs. 2C and 2D). A granular chromosome surface morphology (tens of nanometer corrugations) was typically observed, presumably a consequence of the condensation process of the nucleoprotein fiber during mitosis. In prometaphase the largest corrugation amplitude was visible, whereas in metaphase the chromosome surface appeared smoothly contoured (Figs. 2E and 2F). At higher magnification the most prominent structural features of metaphase chromosomes were the chromatids and the centromere. The latter is composed of parallel bundles of fibrous elements (Wanner et al., 1991; Martin et al., 1996). During condensation the clusters of chromatin fibers may assemble as intermediates in the construction of an axial structure, which is further compacted in the fully condensed (metaphase) chromosome.

\section{A Complex Chromatin Network Remained after Controlled Decondensation of Metaphase Chromosomes}

For revealing structural details of the chromosome substructure, samples of metaphase chromosomes were treated with proteinase $K$ and citrate buffer. In Fig. 3 representative SFM images of chromosomes after proteinase $K$ digestion are shown at various scan sizes. As a prominent consequence of decondensation, chromosomes were flattened on the glass surface. For removal of scan distortions from sample contaminations during SFM imaging (e.g., such as indicated by the arrows in Fig. 3A), a sticky tape was applied to the sample surface without any detectable loss of the chromatin fine structure (Fig. 3B). The sticky tape removed an upper layer of contaminations and was generally applicable for removing weakly adsorbed contaminations of dried samples exposed to air. For comparison, the mean height of (pro)metaphase chromosomes was extracted from SFM images obtained under various experimental conditions and summarized in Table 1 . Within the range of error similar mean heights were obtained for CPD prometaphase and metaphase chromosomes (Table 1, first and second rows). In contrast, decondensed chromosomes appeared to be appreciable 

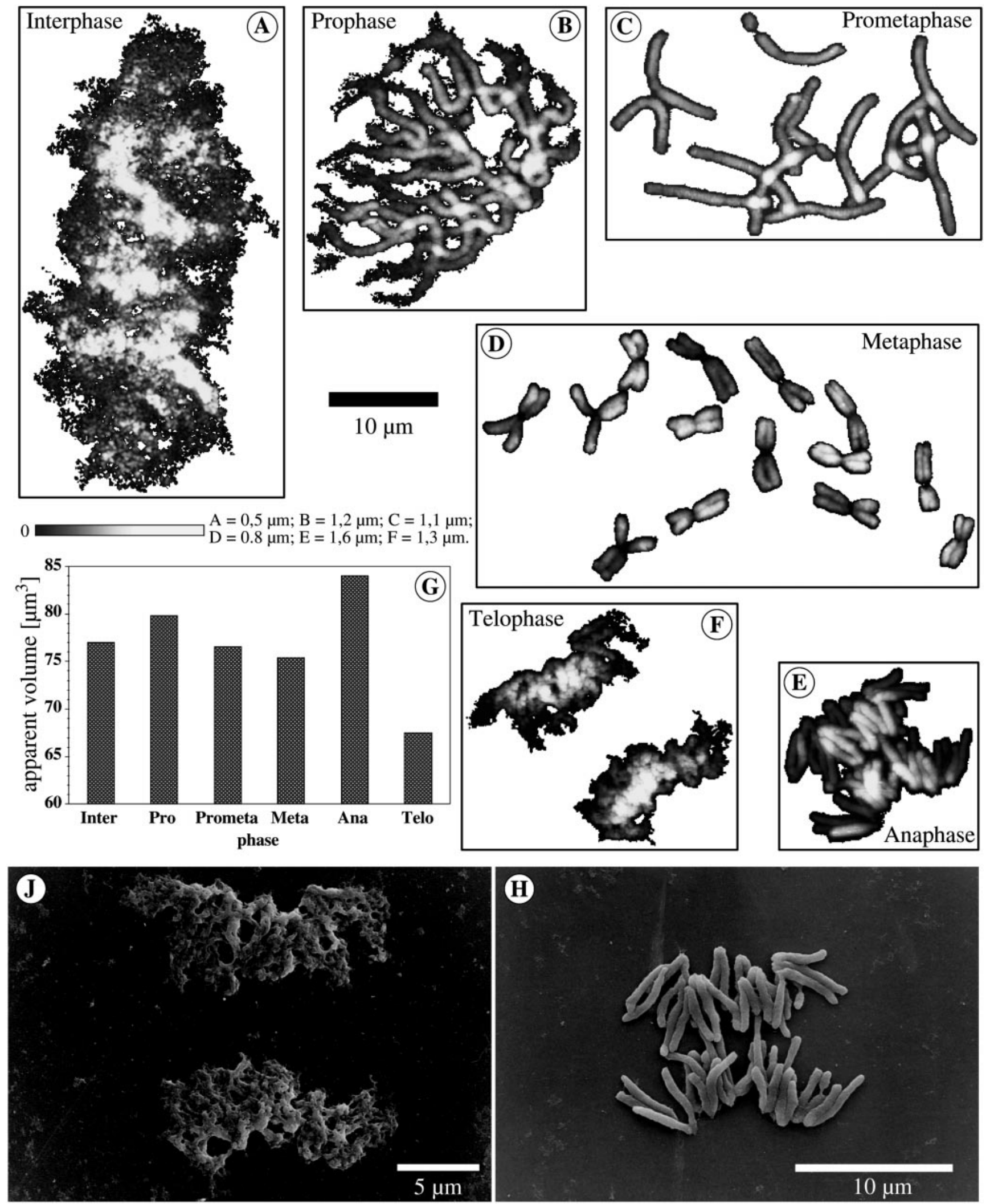

FIG. 1. Different stages of barley mitosis in the SFM after background subtraction and structural filtering of the residue chromatin structure. Normalized lateral dimensions. Sputtered with Au/Pd (coated) and uncoated. (A) Interphase, uncoated. (B) Prophase, coated. (C) Prometaphase, coated. (D) Metaphase, uncoated. (E) Anaphase, coated. (F) Telophase, coated. (G) Apparent volume of the chromatin structures in A-F. The volume calculation based on an image processing algorithm (for details see text). (H) FESEM micrograph of anaphase and ( $\mathrm{J})$ of telophase shown in $\mathrm{E}$ and $\mathrm{F}$, respectively. 

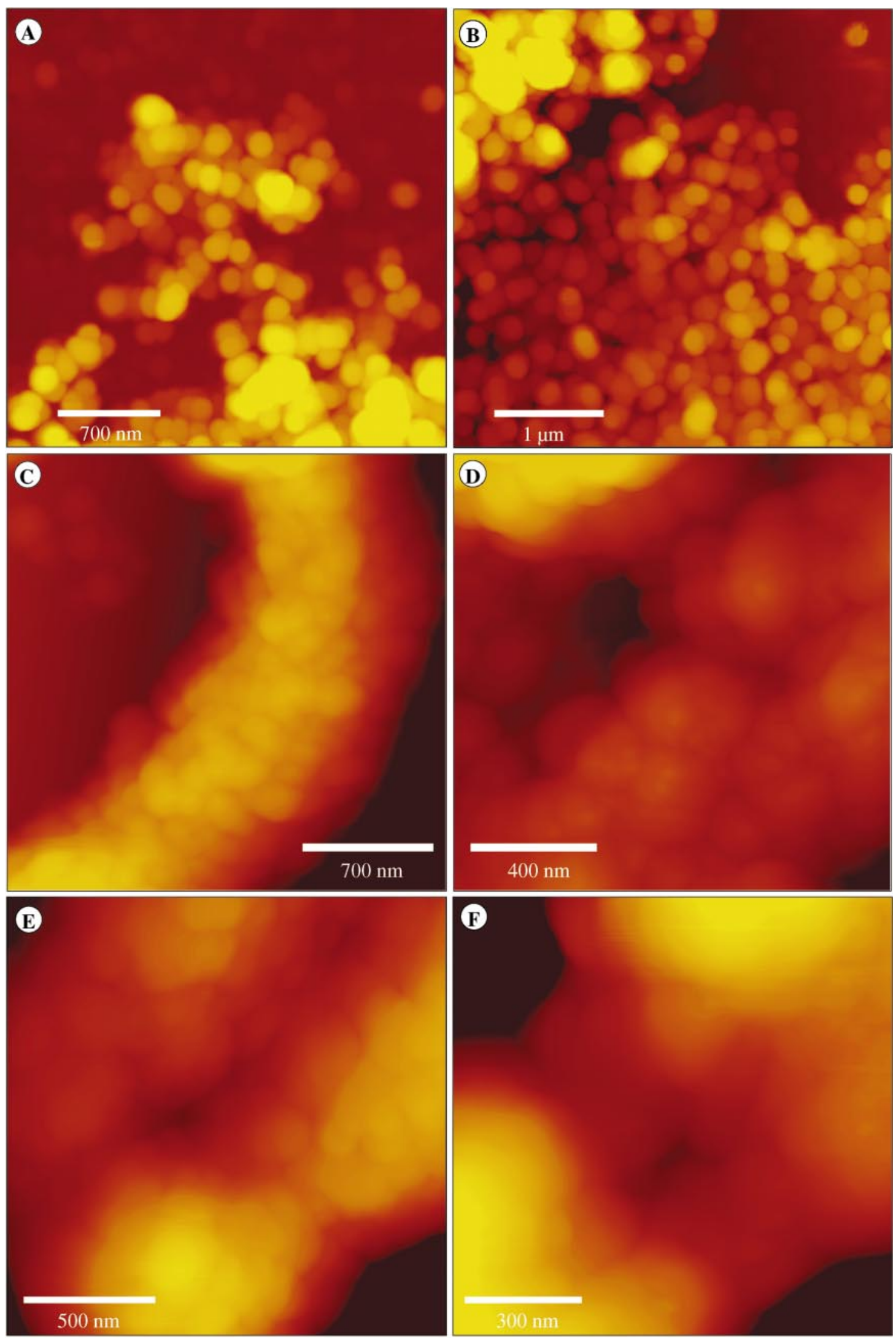

0

$\mathrm{A}=80 \mathrm{~nm} ; \mathrm{B}=120 \mathrm{~nm} ; \mathrm{C}=600 \mathrm{~nm} ; \mathrm{D}, \mathrm{E}=400 \mathrm{~nm} ; \mathrm{F}=700 \mathrm{~nm}$.

FIG. 2. SFM images of selected regions of different states of condensation; uncoated hardware zooms. (A,B) Interphase. Structural features are discernible down to $50 \mathrm{~nm}$ in diameter. (C,D) Prometaphase with corrugated surface. (E,F) Metaphase with smooth surface. In $\mathrm{E}$ a region with a chromatid gap is shown and in $\mathrm{F}$ the centromeric region is visualized. 

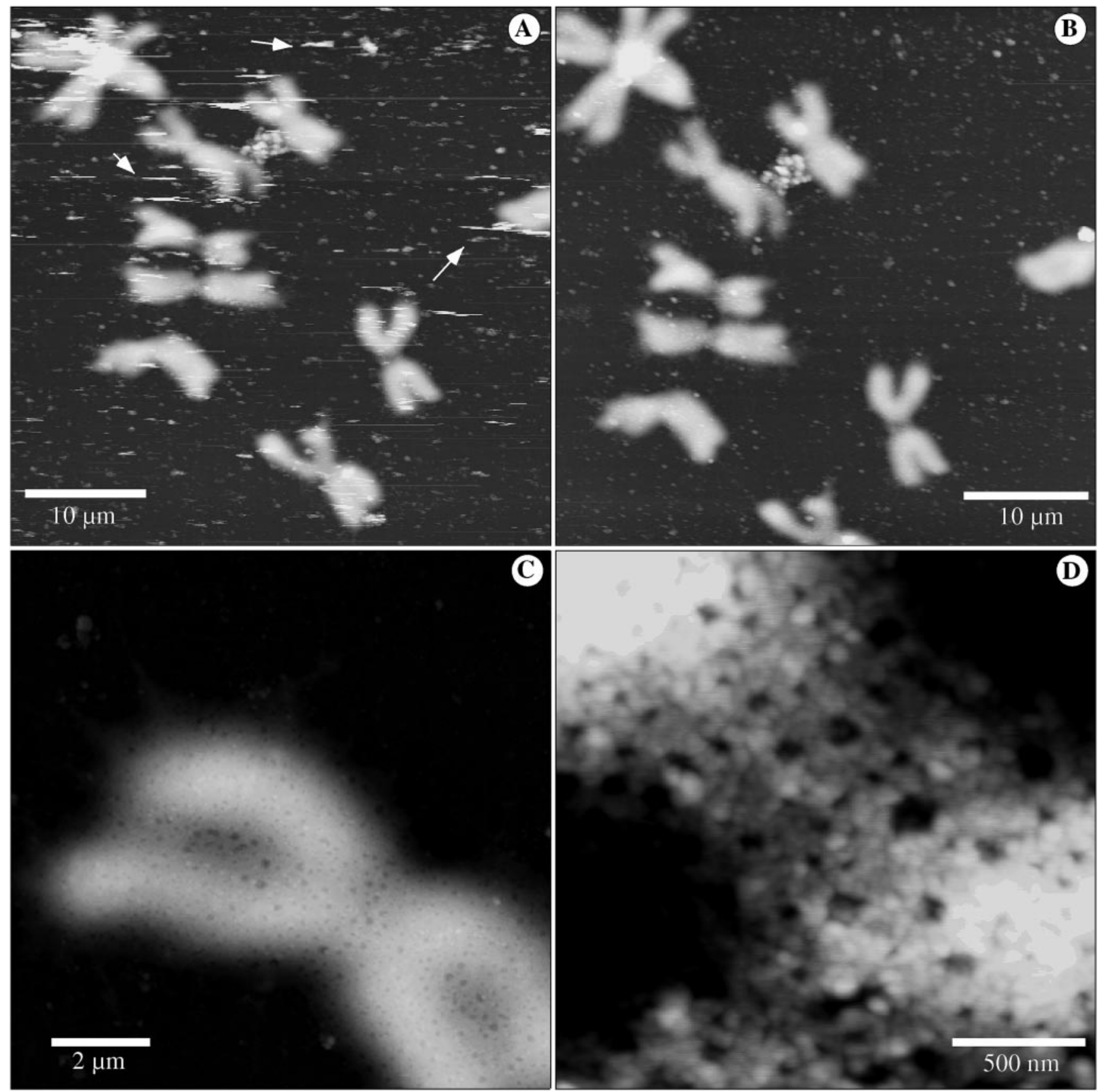

0

$\mathrm{A}, \mathrm{B}=800 \mathrm{~nm} ; \mathrm{C}=600 \mathrm{~nm} ; \mathrm{D}=40 \mathrm{~nm}$.

FIG. 3. SFM imaging of proteinase $K$-treated chromosomes in air. (A) Surface area prior to applying a sticky tape to the surface. Due to sample instabilities typical scan distortions (indicated by the arrowheads) are obvious. (B) Same surface area as in A after the sticky tape was applied. (C) Hardware zoom of an individual chromosome. (D) Hardware zoom of the ultrastructure. A netlike morphology that can be clearly discriminated from the structure of the basal plane is obvious.

lower by a factor of $4-5$ (Table 1 , third row). Thus from Table 1 it is evident that after decondensation by proteinase $K$ treatment the mean height of the chromosomes is drastically reduced compared to the condensed (undigested) structures. In Figs. 3C and $3 \mathrm{D}$ a porous, netlike surface morphology is obvious with a mesh width in the range of $50-100 \mathrm{~nm}$. At high resolution it was revealed that the porous structure was homogeneously distributed over the entire chromosome domain. In a former publication we have shown chromosome structures after impregnation with an organometallic Pt compound (plati- 


\section{TABLE I}

Mean Height of Prometaphase and Metaphase Chromosomes under Various Conditions

\begin{tabular}{lcc}
\hline & Mean height $(\mu \mathrm{m})$ & $\mathrm{SD}(\mu \mathrm{m})$ \\
\hline Prometaphase, CPD & 0.61 & 0.05 \\
Metaphase, CPD & 0.57 & 0.07 \\
Metaphase, proteinase K, CPD & 0.13 & 0.02 \\
Metaphase, in liquid & 1.20 & 0.12 \\
Metaphase, proteinase K, in liquid & 0.37 & 0.07 \\
\hline
\end{tabular}

Note. Data were collected from samples comprising 7 to 14 chromosomes. For consistency, from each sample the upper $10^{4}$ pixels were used for the height statistics including mean and standard deviation (SD).

num blue) for specifically staining DNA(Wanner and Formanek, 1995). In the BSE micrographs of such chromosomes, a netlike DNA pattern is perceptible with morphology similar to that in Fig. 3D. Thus it is conceivable that part of the protein can be removed by proteinase $K$ digestion without changing the overall appearance of the DNA distribution within the chromosome domain.

Chemical decondensation of metaphase chromosomes with citrate buffer was performed in order to investigate the different states of decondensation of the nucleoprotein fiber. In Fig. 4A a metaphase plate is presented after decondensation in the FESEM prior to SFM and in Fig. 4B after SFM imaging. The corresponding SFM surface relief is shown in Fig. $4 \mathrm{E}$. Drastic changes in the FESEM image contrast after SFM are obvious. Since the sample surface is covered by a thin $\mathrm{Au} / \mathrm{Pd}$ layer we must conclude that the SFM tip alters the layer properties during scanning. Consequently, it must be considered that the physicochemical surface properties are modified by tip-sample interaction (e.g., reconstruction and/or mechanical or chemical aging of the metal layer) in an unknown manner. In Figs. 4C and 4D a detailed view of the fine structure in the FESEM before and after SFM imaging, respectively, are shown. After careful inspection of the FESEM and SFM images we conclude that the fine structure of the surface relief is not changed during SFM and we could not detect any loss of surface material during SFM imaging.

Decondensation was initially associated with a gradual elongation and loosening of the chromosome axis. Strikingly, decondensation did not affect the chromosome structure isotropically but seemed to proceed in a bipolar manner starting from the telomeric regions. As loosening progressed, higher order structures (aggregates) became visible, i.e., clusters of nucleoprotein material and fibrous structures of distinct size. The arrangement of the aggregates showed much variation between different metaphase plates and samples. In the final stages of decondensation, aggregates separate and individual chromosomes were no longer recognizable. Figures $4 \mathrm{~F}-4 \mathrm{~K}$ show details of the decondensed chromatin halo at higher resolution in the SFM. The chromatin is dispersed radially from the chromosome domain and is more decondensed in the outer regions of the halo. Inspection of different frames at higher structural resolution revealed a mixture of fibers of various diameters and of granules and voluminous aggregates probably from partially condensed chromatin. Despite the heterogeneous morphology of the decondensed chromatin, selected regions exhibiting filamentous structures were amenable for SFM metrology. Taking into account the broadening of the lateral dimensions by the SFM tip, the apparent width of the various fibers averaged in the size ranges expected for DNA, for the nucleosomal chain, and for higher order chromatin structures such as the 30-nm fiber and 100- to 300-nm fibrous elements. A fiber substructure was not resolvable by SFM. The investigation of the nanometer topography of decondensed chromatin clearly shows the limitations of the applied sample preparation technology including surface contaminations (nanometer particles) and disruption of the chromatin fine structure (disassembly and dissociation of nucleosomes and other DNAassociated proteins).

\section{Chromosomes Prepared by the Drop Technique Are Suitable for SFM Imaging in Liquid}

We imaged chromosomes in the SFM by omitting any intermediate drying step in the drop preparation procedure. The combination of an inverted light microscope with a SFM imaging tool in the BioScope enabled localization and positioning of the chromatin within the scan range of the SFM. The isolated chromosomes, which were not allowed to dry during preparation, retain a $3-\mathrm{D}$ appearance. In general, imaging was delicate since the chromosomes were efficiently removed from the glass support by sharp tips, as was the case with Ultralevers and Hart Probes (tip radius $3-5$ and $\sim 10 \mathrm{~nm}$, respectively) even under minimum tip load. The detachment process could be followed in the light microscope and usually appeared immediately during the first contact (scan line) of the tip with an individual chromosome, which was then removed as a whole from the surface. Imaging with pyramidal shaped $\mathrm{Si}_{3} \mathrm{~N}_{4}$ tips (tip radius $30-50 \mathrm{~nm}$ ) yielded more stable imaging conditions but was accompanied by a decrease in structural resolution. Figure 5A shows a metaphase plate in an aqueous environment in the SFM equipped with a pyramidal shaped tip. The latter exhibited characteristic SFM imaging artifacts. From Fig. 5B at higher resolution it is obvious that the edge profiles of the chromosomes are images of the 

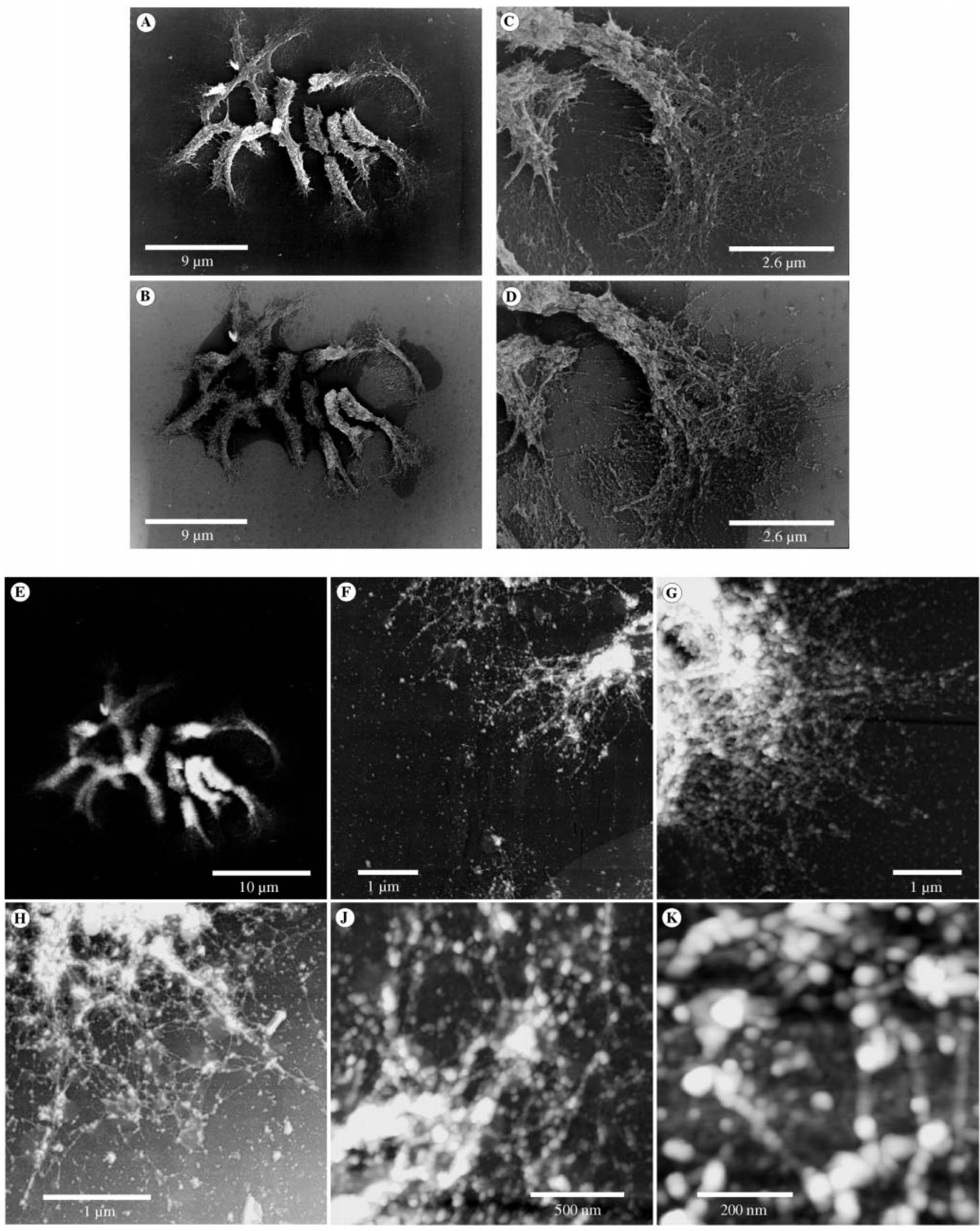
tip profile, thus creating sidewall images of the pyramid at steep surface profiles. Inspection of the surface morphology of the chromosome surface exposed to the top of the pyramid revealed a granular surface structure in qualitative agreement with the CPD dried structure. According to Table 1, the mean height of hydrated chromosomes is about twice the CPD structure, indicating that the native hydrated three-dimensional structure could not be fully preserved during dehydration and/or CPD. We must emphasize that this discrepancy is very mild compared to results of the swelling behavior of rehydrated (former air-dried) chromosomes of human lymphocytes. For those, a factor of 5-7 was typically observed (De Grooth and Putman, 1992; Fritzsche et al., 1994b), indicating a severely collapsed chromosome structure.

In Figs. 5C-5F SFM images of proteinase $K$ treated chromosomes in liquid are presented (cf. Fig. 3 ). The contours of the chromosomes are clearly visible but details of their fine structure were not resolvable (Fig. 5D), probably due to the convolution of topography and elastic response. The apparent mean height of the hydrated chromosome was enlarged two- to threefold, compared with the CPD structure (Table 1), which should be due mainly to the contribution of the hydration behavior of the chromatin. Operating the SFM in tapping mode at minimum tip load was essential for nondestructive imaging of the fragile residue chromosome structure, while permanent contact mode failed due to plastic deformation. The contribution of plasticity and elasticity in the image contrast could be triggered by switching between permanent contact and tapping mode during tip tracking. In Fig. 5E part of the structure was removed in a controlled manner by hardware zooming (see region indicated by the arrow in Fig. 5E) in permanent contact mode prior to imaging in tapping mode. After positioning an appropriate SFM imaging frame (in tapping mode) even an entire chromosome could be easily removed line by line from the glass surface by switching to the permanent contact mode (cf. Figs. $5 \mathrm{C}$ and $5 \mathrm{~F}$, the region indicated by the arrows). These proteinase $K$-treated samples should be attractive for chromosomal microdissection essays; the latter were established by Thalhammer et al. (1997). The behavior of proteinase $K$-treated chromosomes during SFM imaging in liquid indicates that non-DNA material cru- cially contributes to the stiffness of the intact condensed structure in Fig. 5A.

\section{DISCUSSION}

By using unsynchronized barley root tips, snapshots of different states of mitosis were obtained. Changes in the morphology of chromosomes during the different stages of mitosis have been examined by FESEM and SFM. For all chromatin samples investigated by SFM, stable imaging was achieved without any measurable changes of the topography. Our results show that the drop preparation technique originally adapted for FESEM is widely applicable in microscopic investigations of the chromatin structure during mitosis and is amenable for structural investigations/manipulations by SFM in air and in liquid. Prior to sample preparation for microscopy, chemical fixation with ethanol-acetic acid and glutaraldehyde was essential in order to preserve details of the native chromatin structure. For SFM imaging in liquid, chromosomal structures were remarkably stable without any intermediate drying step. In former SFM studies of chromosome structure an intermediate drying step was always essential during sample preparation in order to attain sufficient sample stability and fixation of the rehydrated specimen on the solid support. The investigation of the different states of condensation of the chromatin during mitosis shows that higher resolution by SFM is achievable on flat and dry surfaces, i.e., on chromosome surfaces facing the tip apex (Fig. 2), exhibiting nanometer corrugations, and on decondensed chromatin that spread out on the glass surface (Fig. 4). For the former, the topographic resolution was down to chromomer dimensions and for the latter it was down to the level of the nucleosomal chain and of other fiber classes in chromatin organization.

\section{Limits of Structural Resolution in FESEM and SFM of the Chromosome Structure}

Although much information about chromatin appearance and behavior within the cell has been obtained using light microscopy, greater resolution is needed for a thorough understanding of the chromosome organization. Compared to the instrumental resolution of modern light microscopes of about half the wavelength of the incident light (typically $\sim 250$

FIG. 4. Controlled decondensation of glutaraldehyde-fixed metaphase chromosomes. The drop technique was applied, followed by incubation in citrate buffer, fixation in glutaraldehyde, and CPD. (A) A metaphase plate after decondensation in the FESEM prior SFM imaging and (B) the same area after SFM imaging. (C,D) Zoom of the images shown in A and B before and after SFM, respectively. (E) SFM surface relief of the structure shown in A. (F-K) SFM hardware zooms of selected regions of the decondensed chromatin halo. At higher resolution a complex surface pattern of granules and fibers was obvious. 


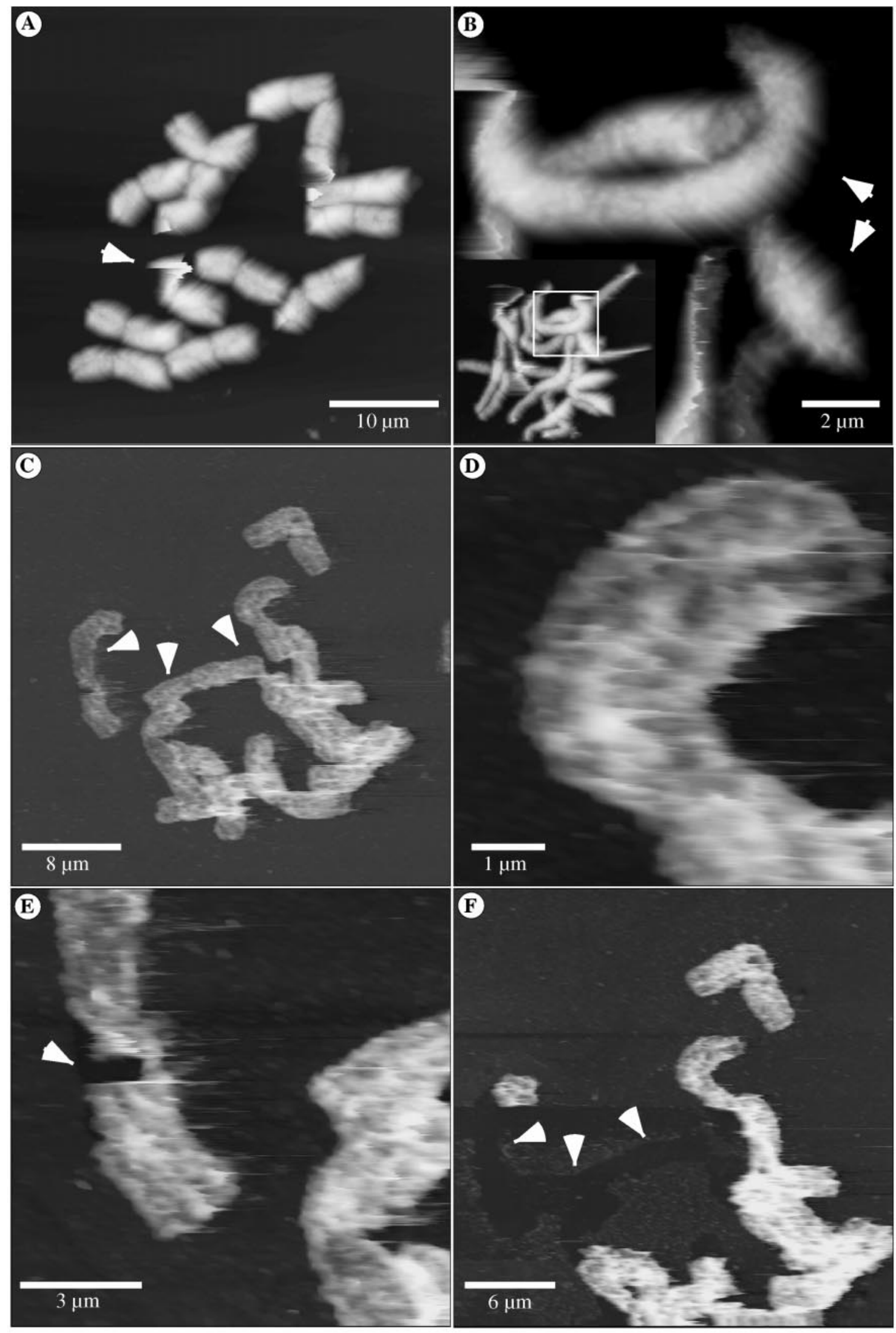

0

$\mathrm{A}=2,7 \mu \mathrm{m} ; \mathrm{B}=1,7 \mu \mathrm{m}$ (Inset $=2,2 \mu \mathrm{m}) ; \mathrm{C}=2 \mu \mathrm{m} ; \mathrm{D}-\mathrm{F}=800 \mathrm{~nm}$. 
$\mathrm{nm}$ ), the resolution power of the FESEM is about 1.5 $\mathrm{nm}$. However, due to sputter coating and restrictions in the exposure time of chromosomes toward the high-energy electron beam, the experimental limit of resolution is typically in the range of 5-10 nm. By means of SFM we could not detect any significant structural alterations introduced by sputter coating of CPD dried samples. In SFM, surface profiling is performed with a stylus of finite sharpness and the resolution capability of the SFM is directly related to the tip geometry. The structural resolution on flat surfaces (with corrugation amplitudes within the tip diameter) is basically limited by the point detection capability of a SFM tip as given by the diameter of its apex. At present, long tips grown in a FESEM from contaminations of the vacuum chamber (EBD tips) are the most suitable tips for imaging steep structures. The batch of Hart Probe tips we used in chromosomal imaging had lengths of about $1.5 \mu \mathrm{m}$ with a tip diameter of roughly $15-20 \mathrm{~nm}$. At steep sidewalls the contact between tip and sample is not restricted to the very top of the tip but also involves its face. From inspection of SFM images of metaphase chromosomes (e.g., in Fig. 1D) there was an obvious structural dilation of $\sim 50 \mathrm{~nm}$ at side walls while surmounting a mean step height of about 0.6 $\mu \mathrm{m}$ from the glass surface to the chromosome top. Assuming a conical tip shape, this is consistent with an apex angle of about $5^{\circ}$, which is an excellent value for these EBD tips and should yield only a minor contribution to the volume calculation. As a disadvantage, imaging in tapping mode with these long EBD tips was not achievable due to large scan instabilities, probably induced by intrinsic bending motions of the tip and amplified by the feedback electronics of the SFM. One reason for the sample instability of chromosomes in liquid using sharp tips (Figs. 5A and $5 \mathrm{~B})$ is the elasticity of the nucleoprotein structure. Sharper tips exert a higher local pressure than blunt end tips (same force constant and cantilever bending). In a former study rehydrated chromosomes exhibited a rubber-like consistency (Fritzsche et al., 1994b). Due to this cohesion of the condensed chromatin, the SFM tip penetrates and sweeps the entire chromosome from the glass surface. Our data indicate that imaging intact chromosomes is a delicate compromise between structural resolution and sample stability.

\section{Structural Hierarchies in Chromosome Organization}

Chromosomes are formed from chromatin in its most condensed state, which is abundant in equal amounts of DNA, histones, and nonhistone proteins. The amount of DNA in the diploid genome of barley is $N_{\mathrm{bp}}=10.7 \times 10^{9} \mathrm{bp}$ (Bennet and Smith, 1976, 1991). The chromosome structure is dominated by the nucleosome ( 8 histones $+140 \mathrm{bp}$ ) particles, each $\sim 5.5 \mathrm{~nm}$ in width $(a$-axis $)$ and $\sim 11 \mathrm{~nm}$ in diameter ( $b$-axis) and linked by $\sim 60 \mathrm{bp}\left(n_{\mathrm{li}}\right)$ DNA on average (Richmond et al., 1984; Kornberg and Klug, 1981). The nucleosome is the repeating unit of the elementary fibril (nucleosomal chain) and is responsible for packing and organizing the DNA in the cell nucleus. The number of nucleosomes per barley genome is $N_{\text {nuc }}=5.35 \times 10^{7}\left(N_{\mathrm{bp}} / 200\right)$. Thus for a chain of nucleosomes with their short $(a)$ axis oriented either parallel or normal to the surface, the contour length of the barley genome is within the range of $0.3\left(N_{\text {nuc }} a\right)-0.6\left(N_{\text {nuc }} b\right)$. That is, for the infinitely dispersed chromatin, i.e., a surface (mono)layer of nucleosomes similar to the chromatin appearance presented by Fritzsche et al. (1994a; Fig. 1), a surface area within $\sim 3000 \mu^{2}\left(a b N_{\text {nuc }}\right)$ and $\sim 5000 \mu \mathrm{m}^{2}(\pi$ $(b / 2)^{2} N_{\text {nuc }}$ ) should be covered by nucleosomes. With this approach the contributions of the linker DNA and nonhistone proteins were ignored. In Fig. 1A the

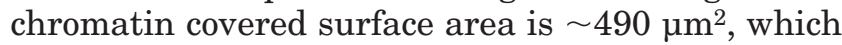
is only $1 / 7$ to $1 / 10$ the area occupied by a nucleosomal (mono)layer. That is, the interphase chromatin in Fig. 1A is not fully dispersed on the level of the nucleosomal chain, which is probably due to incomplete spreading during sample preparation and/or due to the chromatin appearance in a state of partial condensation.

An interesting question is the biological implication of the absolute volume values calculated from the topography of the CPD samples (Fig. 1G). Assuming a cylindrical symmetry of the DNA strand with radius $r=1 \mathrm{~nm}$ and with a rise per basepair for the canonical B-form $h=0.34 \mathrm{~nm}$, the total DNA volume $\left(\pi r^{2} h N_{\mathrm{bp}}\right)$ is $11.4 \mu^{3}$ for the diploid (mitotic) set.

FIG. 5. SFM imaging of chromosomes in liquid prepared without any intermediate drying step during drop sample preparation. (A) Metaphase plate immersed in water and imaged with a pyramidal shaped $\mathrm{Si}_{3} \mathrm{~N}_{4}$ tip. The arrow points to sample instabilities during scanning. (B) Hardware zoom of prometaphase chromosomes within the region indicated by the square in the inset. (Inset) Overview of the prometaphase plate. $\mathrm{Si}_{3} \mathrm{~N}_{4}$ tip. Inset frame size is $33 \times 33 \mu^{2}$. The SFM image exhibits characteristic tip artifacts at steep edges (see arrows), i.e., apparent structural broadening by the tip-sample convolution. (C-F) Metaphase chromosomes after proteinase $K$ treatment in liquid. Tapping mode. Ultralever. (C) Overview of a selected surface area. (D) Hardware zoom of a region in C. The topographic contrast is decreased probably due to elastic contributions of the residue chromosome structure. (E) Imaging and manipulation of the hydrated chromatin by triggering the tip load. As indicated by the arrow, part of the residue chromatin was removed by the tip during imaging in permanent contact mode prior imaging in tapping mode at minimum load. (F) Selective removal of the structure indicated by the errors in $\mathrm{C}$. 
The total volume of the nucleosome core $\left(\pi(b / 2)^{2} a\right.$ $\left.N_{\text {nuc }}\right)$ is $28.0 \mu \mathrm{m}^{3}$ and it is $3.4 \mu \mathrm{m}^{3}\left(\pi r^{2} h n_{\mathrm{li}} N_{\text {nuc }}\right)$ for the linker DNA. Including an equal amount of nonhistone proteins (assuming the nucleosome core is $20.0{\mu m^{3}}^{3}$ ) the total dry volume of a densely packed chromosome plate should be $\sim 51 \mu^{3}$, which is in the range of $60-70 \%$ of the volume extracted from our experimental data on the CPD specimen. From FESEM we know that the chromatin appears porous (due to internal cavities) during mitosis, most prominently in prometaphase (Martin et al., 1996). According to our SFM measurements these internal cavities contribute about $30-40 \%$ to the apparent chromosome volume. Inspection of the lateral dimensions of the dry and hydrated chromosomes (cf. Figs. $1 \mathrm{D}$ and 5) led us to conclude that the glass surface area covered by the chromosomes differed no more than 10-20\%, although the height of the hydrated chromosomes was enlarged by a factor of $\sim 2$ (Table 1 ). That is, the hydrated chromosome plate volume should be in the range of $150-200{\mu m^{3}}^{3}$. In comparison, the radius $\left(r_{\mathrm{n}}\right)$ of the barley nucleus is typically $\sim 5 \mu \mathrm{m}$, corresponding to a volume of $\sim 520 \mu^{3}{ }^{3}(4 / 3$ $\pi r_{\mathrm{n}}^{3}$ ), which is about 2.5-3.5 times the volume required for harvesting the hydrated diploid barley chromatin. According to the present knowledge of the chromosome architecture it is believed that a sister chromatid is organized by a series of looped domains of a single fiber of densely packed nucleosomes. Decondensation of chromosomes either by citrate buffer or by proteinase $K$ treatment as shown in Figs. 4 and $5 \mathrm{C}-5 \mathrm{~F}$, respectively, led to elimination of the structural integrity (unfolding) of the chromosome. After incubation with citrate buffer, fibrous substructures became obvious; they are believed to constitute intermediate structures during chromatin condensation in nearly all of the chromatin packing models (discussed in Cook, 1995). The citrateinduced decondensation process was on a time scale of minutes, and the amount of chromatin dispersed in the halo varied from sample to sample. Most probably, the latter observation is related to sample history. In this context, one must keep in mind that in contrast to the preparation of the "snapshots" of mitosis (Fig. 1) the chromosome decondensation is induced under the constraint of the surface immobilization. In the initial step of decondensation, the intramolecular interactions are decreased followed by chromatin dispersion in the telomeric and centromeric regions (Wanner and Formanek, unpublished results). Such a behavior correlates with a structural hierarchy of chromatin organization. Since we were able to prepare chromatin of different levels of decondensation, we conclude that dehydration and CPD influence only the appearance of the dissociated amount of chromatin, i.e., the chromatin halo.
It is not clear whether the chromatin halo in Fig. 4 was generated by diffusional processes (driven by osmotic pressure) followed by adsorption or induced by the interfacial forces during fluid exchange. However, the partially disrupted chromatin topography in Fig. 4 indicates the importance of an appropriate control of the kinetics and of the binding affinities during sample preparation. Our results on the influence of the CPD, hydration, citrate buffer, and proteinase $K$ treatment on chromosome manifestation demonstrate the importance of adequate chromatin preparation and of the necessity of combining different microscopic techniques in revealing the biofunctionality of these structures. In this context, we have shown that the drop method for chromatin spreading provides specimens suitable for FESEM and for SFM imaging in air and in liquid. Morphological features of the isolated mitotic chromatin are retained, enabling the investigation and manipulation of its structure. Further refinement of the preparation technique should provide specimens for direct in vitro SFM observations of the structural changes induced by chemical and/or enzymatic compounds under physiological conditions.

We thank W. Fritzsche for valuable discussions and S. Steiner for excellent technical assistance. This work was supported by the Max Planck Society and the German Research Council (DFG Grants Jo 105/9 and WA 603/2) to T.M.J. and G.W.

\section{REFERENCES}

Allen, M. J., Dong, X. F., O’Neill, T. E., Yau, P., Kowalczykowski, S. C., Gatewood, J., Balhorn, R., and Bradbury, E. M. (1993) Atomic force microscope measurements of nucleosome cores assembled along defined DNA sequences, Biochemistry 32, 8390-8396.

Allen, M. J., Lee, J. D., Lee, C., and Balhorn, R. (1996) Extent of sperm chromatin hydration determined by atomic-force microscopy, Mol. Reprod. Dev. 45, 87-92.

Barrett, S. (1997) ImageSXM software (version 1.61-7) (Home page/download: http://reg.ssci.liv.ac.uk/).

Bennet, M. D., and Smith, J. B. (1976) Nuclear DNA amount in angiosperms, Philos. Trans. R. Soc. London B 274, 227-274.

Bennet, M. D., and Smith, J. B. (1991) Nuclear DNA amount in angiosperms, Philos. Trans. R. Soc. London B 334, 309-345.

Busch, W., Martin, R., and Herrmann, R. G. (1994) Sensitivity enhancement of fluorescence in situ hybridization on plant chromosomes, Chromosome Res. 2, 15-20.

Cook, P. R. (1995) A chromomeric model for nuclear and chromosome structure, J. Cell Sci. 108, 2927-2935.

De Grooth, B. G., Putman, C. A. J., van der Werf, K. O., van Hulst, N. F., van Oort, G., and Greve, J. (1992) Chromosome structure investigated with the atomic force microscope, SPIE Scanning Probe Microsc. 1639, 205-211.

De Grooth, B. G., and Putman, C. A. J. (1992) High-resolution imaging of chromosome-related structures by atomic force microscopy, J. Microsc. 168, 239-247.

Dolezel, J., Cihalikova, J., and Lucretti, S. (1992) A high-yield procedure for isolation of metaphase chromosomes from root tips, Vicia faba L. Planta 188, 93-98. 
Fritzsche, W., Schaper, A., and Jovin, T. M. (1994a) Scanning force microscopy of chromatin in air and in liquid, Scanning 17, 148-155.

Fritzsche, W., Schaper, A., and Jovin, T. M. (1994b) Probing chromatin with the scanning force microscope, Chromosoma 103, 231-236.

Fritzsche, W., and Henderson, E. (1996) Volume determination of human metaphase chromosomes by scanning force microscopy, Scanning Microsc. 10, 103-110.

Harrison, C. J., Allen, T. D., Britch, M., and Harris, R. (1982) High-resolution scanning electron microscopy of human metaphase chromosomes, Cell Sci. 56, 409-422.

Harrison, C. J., Jack, E. M., and Allen, T. D. (1987) Light- and scanning electron microscopy of the same metaphase chromosomes, in M. A., Hayat (Ed.), Correlative Microscopy in Biology: Instrumentation and Methods, pp. 187-248. Academic Press, New York.

Kornberg, R. D., and Klug, A. (1981) The nucleosome, Sci. Am. 244, 52-64.

Leuba, S. H., Yang, G. L., Robert, C., Samori, B., Vanholde, K., Zlatanova, J., and Bustamante, C. (1994) 3-Dimensional structure of extended chromatin fibers as revealed by tapping-mode scanning force microscopy, Proc. Natl. Acad. Sci. USA 91, 11621-11625.

Marsden, M. P. F., and Laemmli, U. K. (1979) Metaphase chromosome structure: Evidence for a radial loop model, Cell 17, 849-858.

Martin, R., Busch, W., Herrmann, R. G., and Wanner, G. (1994) Efficient preparation of plant chromosomes for high-resolution scanning microscopy, Chromosome Res. 2, 411-415.

Martin, R., Busch, W., Herrmann, R. G., and Wanner, G. (1996) Changes in chromosome ultrastructure during the cell cycle, Chromosome Res. 4, 288-294.

McMaster, T. J., Hickish, T., Min, T., Cunningham, D., and Miles, M. J. (1994) Application of scanning force microscopy to chromosome analysis, Cancer Genet. Cytogenet. 76, 93-95.

Mullinger, A. M., and Johnson, R. T. (1987) Scanning electron microscope analysis of structural changes and aberrations in human chromosomes associated with the inhibition and reversal of inhibition of ultraviolet light induced DNA repair, Chromosoma 96, 39-44.

Pelling, C., and Allen, T. D. (1993) Scanning electron microscopy of polytene chromosomes (I), Chromosome Res. 1, 221-237.

Pietrasanta, L. I., Schaper, A., and Jovin, T. M. (1994) Imaging subcellular structures by scanning force microscopy, J. Cell Sci. 107, 2427-2437.

Pietrasanta, L. I., Schaper, A., and Jovin, T. M. (1996) Imaging the electrocyte from Torpedo marmorata by scanning force microscopy, Scanning Microsc. 10, 963-974.

Putman, C. A. J., De Grooth, B. G., Wiegant, J., Raap, A. K., Van der Werf, K. O., Van Hulst, N. F., and Greve, J. (1993) Detection of in situ hybridization to human chromosomes with the atomic force microscope, Cytometry 14, 356-361.

Rasch, P., Wiedemann, U., Wienberg, J., and Heckl, W. M. (1993) Analysis of banded human chromosomes and in situ hybridization patterns by scanning force microscopy, Proc. Natl. Acad. Sci. USA 90, 2509-2511.

Rattner, J. B., and Lin, C. C. (1987) The higher order structure of centromere, Genome 29, 588-593.

Richmond, T. J., Finch, J. T., Rushton, B., Rhodes, D., and Klug, A. (1984) Structure of the nucleosome core particle at $7 \AA$ resolution, Nature 311, 532-537.

Rizzoli, R., Rizzi, E., and Falconi, M. (1994) High resolution detection of uncoated metaphase chromosomes by means of field emission scanning electron microscopy, Chromosoma 103, 393-400.

Sumner, A. T. (1991) Scanning electron microscopy of mammalian chromosomes from prophase to telophase, Chromosoma 100, 410-418.

Takayama, S., and Hiramatsu, H. (1993) Scanning electronmicroscopy of the centromeric region of 1-cell chromosomes after treatment with Hoechst-33258 combined with 5-bromodeoxyuridine, Chromosoma 102, 227-232.

Thalhammer, S., Stark, R. W., Muller, S., Wienberg, J., and Heckl, W. M. (1997) The atomic-force microscope as a new microdissecting tool for the generation of genetic probes, J. Struct. Biol. 119, 232-237.

Van Holde, K. E. (1988) Chromatin, Springer-Verlag, New York/ Berlin.

Wanner, G., Formanek, H., Martin, R., and Herrman, R. G. (1991) High resolution scanning electron microscopy of plant chromosomes, Chromosoma 100, 103-109.

Wanner, G., and Formanek, H. (1995) Imaging of DNA in human and plant chromosomes by high-resolution scanning electron microscopy, Chromosome Res. 3, 368-374.

Zentgraf, H., Bock, C.-T., and Schrenk, M. (1987) Chromatin spreading, in Sommerville and U. Scheer (Eds.), Electron Microscopy in Molecular Biology, pp. 81-100. IRL Press, Oxford. 